\title{
Neural Underpinnings of Gesture Discrimination in Patients with Limb Apraxia
}

\author{
Mariella Pazzaglia, ${ }^{1,2}$ Nicola Smania, ${ }^{3}$ Elisabetta Corato, ${ }^{3}$ and Salvatore Maria Aglioti ${ }^{1,2}$ \\ ${ }^{1}$ Dipartimento di Psicologia, Università di Roma "La Sapienza", 00185 Rome, Italy, ${ }^{2}$ Fondazione Santa Lucia, Istituto di Ricovero e Cura a Carattere \\ Scientifico, 00100 Rome, Italy, and ${ }^{3}$ Centro di Rieducazione Funzionale del Policlinico Giambattista Rossi e Scuola di Specializzazione in Medicina Fisica e \\ Riabilitazione, Università di Verona, 37134 Verona, Italy
}

Limb apraxia (LA), is a neuropsychological syndrome characterized by difficulty in performing gestures and may therefore be an ideal model for investigating whether action execution deficits are causatively linked to deficits in action understanding. We tested 33 left brain-damaged patients and 8 right brain-damaged patients for the presence of the LA. Importantly, we also tested all the patients in an ad hoc developed gesture recognition task wherein an actor performs, either correctly or incorrectly, transitive (using objects) or intransitive (without objects) meaningful conventional limb gestures. Patients were instructed to judge whether the observed gesture was correct or incorrect. Lesion analysis enabled us to evaluate the relationship between specific brain regions and behavioral performance in gesture execution and gesture comprehension. We found that LA was present in 21 left brain-damaged patients and it was linked to frontal and parietal lesions. Moreover, we found that recognition of correct execution of familiar gestures performed by others was more impaired in patients with LA than in nonapraxic patients. Crucially, the gesture comprehension deficit correlated with damage to the opercular and triangularis portions of the inferior frontal gyrus, two regions that are involved in complex aspects of action-related processing. In contrast, no such relationship was observed with lesions centered on the inferior parietal cortex. The present findings suggest that lesions to left frontal regions that are involved in planning and performing actions are causatively associated with deficits in the recognition of the correct execution of meaningful gestures.

Key words: limb apraxia; gesture execution; gesture recognition; frontoparietal circuits; brain damage; mirror systems

\section{Introduction}

Limb apraxia (LA) comprises various higher-order motor disorders characterized by the inability or difficulty in performing a particular class of skilled, purposeful limb movements known as gestures. LA is more commonly associated with left frontal and parietal brain damage than with right brain damage (Haaland et al., 2000; Hanna-Pladdy et al., 2001; McClain and Foundas, 2004) and is not explained by elemental motor or sensory systems or by defects in language comprehension, and it typically affects both the ipsilesional and the contralesional limbs. Conceptual and production components of gestural organization may be differentially affected and may lead to ideational and ideomotor apraxia. Although ideational apraxia is linked to defective action and object-use knowledge, ideomotor apraxia is characterized by spatiotemporal errors in gesture pantomime and imitation (Leiguarda and Marsden, 2000). Apraxic deficits are detected by testing the patients' ability to imitate or recognize gestures or to pantomime familiar, object-related gestures in response to com-

\footnotetext{
Received Sept. 24, 2007; accepted Jan. 21, 2008

This work was supported by grants from the Ministero Istruzione Università e Ricerca (PRIN) and Fondo Investimenti per la Ricerca di Base, Italy (RBNE01SZB4) (both awarded to S.M.A.).

Correspondence should be addressed to either Salvatore Maria Agliot or Mariella Pazzaglia, Dipartimento di Psicologia, Università di Roma "La Sapienza," Via dei Marsi 78, 00185 Rome, Italy. E-mail: salvatoremaria.aglioti@uniroma1.it or mariella.pazzaglia@uniroma1.it.

D0I:10.1523/JNEUROSC1.5748-07.2008

Copyright $\odot 2008$ Society for Neuroscience $\quad 0270-6474 / 08 / 283030-12 \$ 15.00 / 0$
}

mand or to the sight of objects (Haaland et al., 2000; Daprati and Sirigu, 2006). Patients with apraxia may be impaired in executing both gestures that involve the use of objects (transitive), such as hammering a nail, and gestures not involving object use (intransitive), such as the hitchhiking sign (Cubelli et al., 2000). Although the impairment of meaningful (e.g., waving goodbye) and meaningless (e.g., moving a hand) gestures may be doubly dissociated (Goldenberg and Hagmann, 1997; Bartolo et al., 2001), apraxic patients often exhibit deficits in both types of gestures (Toraldo et al., 2001; Leiguarda, 2005), at least when presented in an intermingled list (Tessari et al., 2007).

The discovery in the monkey frontal and parietal cortices (Gallese et al., 1996; Fogassi et al., 2005) of complex double-duty neurons that are activated during both action execution and observation (mirror neurons), hints at very tight links between the perceptual and the motor components of an action. In the 1980s, a few pioneering studies in patients with LA reported an association between the inability to perform gestures and understand their meaning, and left parietal lesions (Heilman et al., 1982; Rothi et al., 1985; Watson et al., 1986). However, given the limited power of lesion analysis techniques at that time, no clear lesion mapping was provided. Two recent studies on this issue yielded controversial results. Halsband et al. (2001) reported that patients with left parietal and frontal lesions were impaired in gesture execution but not in gesture comprehension. In contrast, Buxbaum et al. (2005) tested left brain-damaged patients and 


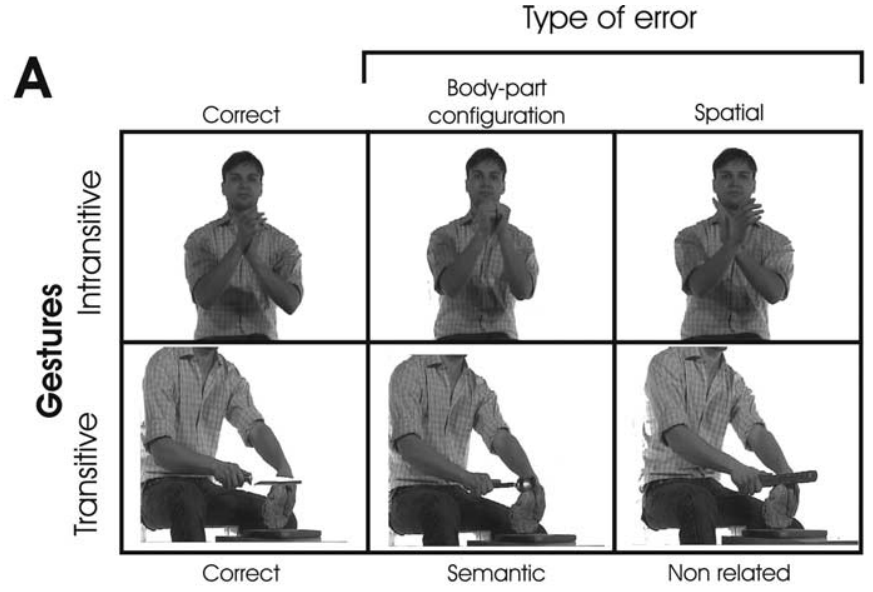

B

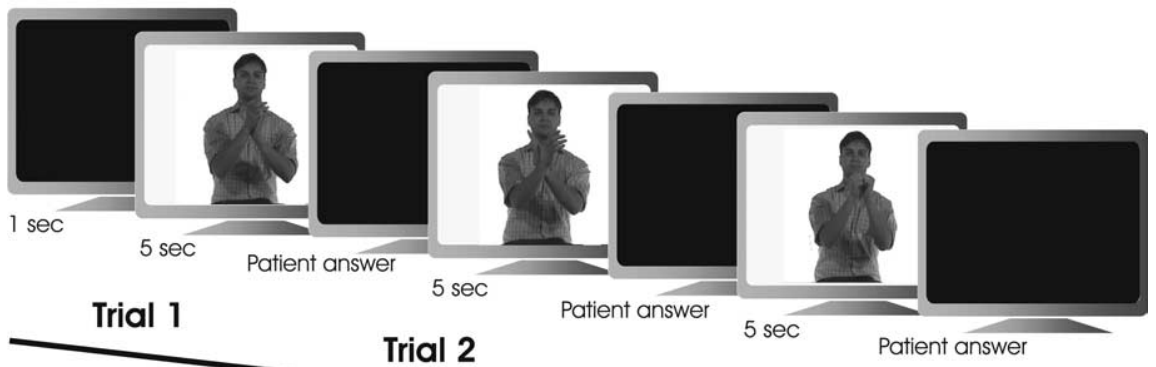

Trial 3 was assigned when the imitation was incorrect in all attempts. Patients with a total score of $<53$ were assigned to the LA group. In the second test, patients were requested to perform seven complex actions that required the use of real objects (hammer; toothbrush; scissors; pistol; pencil eraser; padlock and key; and candlestick holder, candle, and a matchbox). For each action, a correct performance was scored 2, an inaccurate performance in which the action was recognizable but not entirely correct was scored 1 , and an incorrect performance was scored 0 . Total scores $<14$ indicated apraxia. All tests were administered by an expert clinical neuropsychologist who was blinded to the study aims.

Twenty-one LBD patients failed at least one of the two above-described tests and were assigned to the LA $\left(\mathrm{LA}+{ }_{(\mathrm{LBD})}\right)$ group, whereas the remaining patients were assigned to the control $(\mathrm{LA}-)$ group $\left(\mathrm{LA}-{ }_{(\mathrm{LBD})}, 12\right.$ patients; LA- ${ }_{(\mathrm{RBD})}, 8$ patients). The three groups were matched in age (mean $\pm \mathrm{SD}, \mathrm{LA}+_{(\mathrm{LBD})}, 63.7 \pm$ 13 years; $\mathrm{LA}_{(\mathrm{LBD})}, 63.8 \pm 11.4$ years; $\mathrm{LA}^{-(\mathrm{RBD})}, 64.2 \pm 13.3$ years) and education $\left(\right.$ mean $\pm \mathrm{SD}, \mathrm{LA}+{ }_{(\mathrm{LBD})}, 9.5 \pm 4.8$ years; $\mathrm{LA}_{(\mathrm{LBD})}, 9.3 \pm 4.9$ years; $\mathrm{LA}_{(\mathrm{RBD})}, 9.7 \pm 3.5$ years). The interval between the onset of stroke and the time of testing did not differ between the three groups $\left(\mathrm{LA}+_{(\mathrm{LBD})}, 57 \pm 29 \mathrm{~d}\right.$; $\mathrm{LA}-_{(\mathrm{LBD})}, 59 \pm 28 \mathrm{~d}$; and $\mathrm{LA}-{ }_{(\mathrm{RBD})}, 52 \pm$ $23 \mathrm{~d}$ ). Moreover, the performance in the Raven 47 colored progressive matrices (PM47) (Raven et al., 1988), a nonverbal general intelligence test, did not differ significantly in the three groups (mean $\pm \mathrm{SD}, \mathrm{LA}+_{(\mathrm{LBD})}, 21.14 \pm 4.8$; $\mathrm{LA}_{(\mathrm{LBD})}, 25.25 \pm 7.3$; $\left.\mathrm{LA}_{(\mathrm{RBD})}, 23.50 \pm 5.5\right)$.

Noncontextual language comprehension was assessed using the 50-item version of the Token

reported a strong relationship between imitation and recognition of transitive gestures and the association of gesture recognition deficits with lesions in parietal areas, but not in the frontal areas.

In the present study, we devised a novel gesture discrimination test to determine whether the inability to recognize gestures is an essential feature of LA. Furthermore, we used advanced lesion-mapping procedures to assess whether lesions in specific regions of the frontoparietal network that underlies action production are causatively associated with action comprehension deficits.

\section{Materials and Methods}

Forty-one patients suffering from ischemic or hemorrhagic stroke were recruited from the Istituto di Ricovero e Cura a Carattere Scientifico Fondazione Santa Lucia (Rome, Italy) and from the Centro di Rieducazione Funzionale, Policlinico Borgo-Roma (Verona, Italy). Thirty-three patients presented with left brain damage (LBD), and eight patients had right brain damage (RBD). All participants provided written informed consent, and the procedures were approved by the local ethics committee and were in accordance with the ethical standards of the 1964 Declaration of Helsinki. All patients were right-handed according to the Edinburgh Handedness Inventory (Oldfield, 1971).

Neuropsychological assessment. The presence of apraxia was ascertained by two gesture production tests that aimed at testing ideomotor (IMA) (De Renzi et al., 1980) and ideational (IA) (De Renzi and Lucchelli, 1988) apraxia. In the first test, patients were required to use their ipsilesional hands, to imitate 24 finger and hand intransitive configurations (12 meaningless and 12 meaningful) as shown to them by an examiner. In each trial, patients were allowed up to three attempts to imitate the movement correctly. Each attempt was scored 3, 2, or 1 when it was correct on the first, second, or third attempt, respectively. A score of 0 test from the Italian version of the Aachener Aphasie Test (Luzzatti et al., 1996). A one-way ANOVA indicated a significant main effect for group $\left(F_{(2,38)}=25.60 ; p<0.01\right)$. Post hoc analysis indicated that LA+ ${ }_{(\mathrm{LBD})}$ (mean $\pm \mathrm{SD}, 40.6 \pm 12.7$ ) patients were more impaired than LA- ${ }_{(\mathrm{LBD})}$ (mean \pm SD, $56.25 \pm 10.84$ ) patients, who in turn were less accurate than $\mathrm{LA}^{-(\mathrm{RBD})}($ mean $\pm \mathrm{SD}, 71.87 \pm 1.81$ ) patients (all values of $p<0.001$ ). None of the patients presented with visual discrimination deficits (see below) or with signs of unilateral spatial neglect [assessed according to Pizzamiglio et al. (1989)].

Recognition of gestures performed by a model. The ability to recognize gestures performed by a model as correct or incorrect was assessed by an ad hoc devised test. Patients were shown 60 video clips in which an actor performed transitive $(n=30)$ or intransitive $(n=30)$, meaningful conventional limb gestures either correctly or incorrectly. No sound cues the video clip or by the examiner at any time. Ten transitive gestures demonstrating the actual use of the objects (e.g., playing guitar) and 10 intransitive gestures demonstrating meaningful conventional sign (e.g., the hitchhiking) were correctly performed. Incorrect gestures were produced by modifying the correct gestures. Video clips of incorrect transitive gestures were created by changing the object in a given correct gesture (e.g., playing guitar) with an object that was inappropriate for the particular action. The inappropriate object could be semantically related (e.g., flute) or unrelated (e.g., broom) to the correct object (e.g., guitar). Video clips of incorrect intransitive gestures were created by asking the actor to perform a given gesture (e.g., hitchhiking) either by changing the hand or finger configuration crucial for correctly identifying the gesture (e.g., hitchhiking performed by extending the little finger instead of the thumb) or by changing the spatial position of the involved body part (e.g., hitchhiking performed with hand above the head). For each cateregarding correct or incorrect gesture demonstrations were provided by 
gory, 10 incorrect gestures were presented. Additional examples of correct and incorrect gestures are shown in Figure 1.

Procedure. The patients were seated at a distance of $\sim 50 \mathrm{~cm}$ from a 17 inch computer monitor and were requested to observe $5 \mathrm{~s}$ video clips showing meaningful gestures that could be performed correctly or incorrectly. Subjects were asked to simply judge whether the presented gesture was performed in a correct or incorrect way by responding "yes/no" or "wrong/correct." Complex verbal responses or pantomimes of the observed gesture were not allowed. After each clip, patients provided their response within a $5 \mathrm{~s}$ interval. Two types of responses were scored as correct and were assigned 1 point, namely, "yes/correct" responses to gestures performed correctly (hits) and "no/wrong" responses to gestures performed incorrectly (correct rejections). Two types of responses were scored as incorrect and were assigned 0 points, namely, "no/wrong" responses to gestures performed correctly (misses) and "yes/correct" responses to gestures performed incorrectly (false alarms). This procedure enabled us to delineate the contribution of sensitivity and response criterion in determining failures in the test (Green and Swets, 1966).

Each patient was subjected to six practice trials with gestures different from those used during the experimental phase. The patients were provided with feedback on their performance during the practice trials and not in the experimental phase. The order of the correct/incorrect and transitive/intransitive gestures was randomized. In this test, the performance of a group of 20 healthy participants (11 men), matched in age (mean \pm SD, $60.9 \pm 9.4$ years; range, $45-79$ years) and education ( $8.4 \pm$ 2.4 years; range, 5-18 years) with the brain-damaged group, was errorless.

The patients also performed an object identification task, wherein the experimental stimuli, selected from the set of Snodgrass and Vanderwart (1980), depicted common objects (tools, vehicles, musical instruments, household items, and clothing/accessories). In a set of eight object pictures, the patients were asked to indicate the one named by the examiner. This test was performed to rule out the possibility that any impairment in recognizing the correctness of the transitive gestures was attributable to object recognition deficits.

Lesion mapping. The analysis of lesioned regions was based on magnetic resonance imaging (MRI) or computerized tomography scans. T1weighted and T2-weighted MRI data sets were acquired for 35 of the patients. MRI was performed using a $1.5 \mathrm{~T}$ system (Vision; Siemens Medical Systems, Erlangen, Germany). The imaging protocol included the following sequences: (1) conventional T1-weighted turbo spin-echo images [repetition time (TR)/echo time (TE)/excitations/flip angle, 650/ 14/2/70; matrix, $256 \times 256$; in-plane resolution, $0.9 \times 0.9 \mathrm{~mm}$ ] and (2) double-echo turbo spin-echo proton density and T2-weighted images (TR/TE1/TE2/excitations, 3800/22/90/1; matrix, $256 \times 256$; in-plane resolution, $0.9 \times 0.9 \mathrm{~mm})$. Computerized tomography scans were used for the remaining six patients (three $\mathrm{LA}^{-}{ }_{(\mathrm{LBD})}$ and three $\left.\mathrm{LA}-{ }_{(\mathrm{RBD})}\right)$. LA was present only in LBD patients; therefore, the lesion mapping analysis focused on these patients. Using the MRIcro software available at http:// www.sph.sc.edu/comd/rorden/mricro.html (Rorden and Brett, 2000), the lesions were mapped by one experimenter who ignored the test results and the clinical features of the patients. The lesions were drawn manually on slices of a T1-weighted template MRI scan from the Montreal Neurological Institute (MNI) (http://www.bic.mni.mcgill.ca/cgi/ icbm_view). This template is oriented to approximately match Talairach space (Talairach and Tournoux, 1988) and is distributed with MRIcro. The template scan provides various anatomical landmarks for precisely plotting the size and localization of the lesions. The area of brain lesion of each LBD patient was superimposed onto the T1 template to calculate the total brain lesion volume (in cubic centimeters) using MRIcro. The lesion volume was comparable among the three groups (mean $\pm \mathrm{SD}$, $\mathrm{LA}+{ }_{(\mathrm{LBD})}, 35.91 \pm 32.43 \mathrm{~cm}^{3} ; \mathrm{LA}-{ }_{(\mathrm{LBD})}, 34.2 \pm 29.42 \mathrm{~cm}^{3}$; and LA- ${ }_{(\mathrm{RBD})}, 38.2 \pm 30.42 \mathrm{~cm}^{3}$; Kruskal-Wallis test, $H=0.96, p=0.61$ ). We identified regions that may exhibit dysfunction through two different types of anatomical analyses, namely, lesion subtraction analysis [for details, see Rorden and Karnath (2004)] and region-based statistical analysis. Lesion subtraction plots directly compared patients showing the disorder of interest (a lesion overlay with positive values) to a control group (a lesion overlay with negative values). The relative incidence of damage to regions unrelated to the disorder of interest should be equally represented in both patient groups, and therefore should not be highlighted in the subtraction plots. Lesion subtractions were based on proportional values derived from the difference in the number of patients in each group. A region-based statistical analysis was performed on the data using an automated anatomical labeling (AAL) procedure. This procedure was based on the macroscopic anatomical parcellation of the MNI single-subject brain (Tzourio-Mazoyer et al., 2002). The percentage of each damaged brain region with respect to each AAL was computed in each patient.

The statistical significance of the occurrence of a brain lesion was determined using three tests: (1) a $\chi^{2}$ test (with Yates' correction) that allowed us to assess whether, for each group, the number of patients who present a lesion in a given AAL is significantly different. This test was performed when at least $30 \%$ of the sample size presented a lesion in a given AAL region; (2) a Mann-Whitney voxel-count $U$ test that allowed us to assess whether, for each group, the percentage of lesioned tissue in each AAL is significantly different; (3) a voxel-by-voxel test ( $\chi^{2}$ test with Yates' correction) that considered only cortical voxels belonging to the regions that passed one of the previous two tests.

The association of lesional sites with continue behavioral performance in the gesture recognition tasks was assessed by using a specific tool that is freely available online at http://www.sph.sc.edu/comd/rorden/ mricro.html and does not require any patients classification into specific groups (Rorden et al., 2007). Scores in transitive gesture recognition (TGR) and intransitive gesture recognition (IGR) tests and lesion reconstructions in the group of 33 LBD patients were entered in a nonparametric permuted Brunner-Munzel rank-order statistic analysis for each voxel of the brain. The $\alpha$ level was set at $p<0.05$. The levels of significance were corrected for multiple comparisons by using the false discovery rate (FDR) threshold (Nichols and Hayasaka, 2003).

\section{Results}

Twenty-one LBD patients were assigned to the LA+ ${ }_{(\mathrm{LBD})}$ group, based on performance in the IMA (mean, 38.95) and IA (mean, 11.47 ) production tests. The remaining 20 patients were assigned to the LA - group $\left(12 \mathrm{LA}^{-}{ }_{(\mathrm{LBD})}\right.$ : IMA, 65.83; IA, 14; $8 \mathrm{LA}^{-}{ }_{(\mathrm{RBD})}$ : IMA, 68.12; IA, 14).

Maps of overlapping lesions of $\mathrm{LA}+_{(\mathrm{LBD})}$ and $\mathrm{LA}-{ }_{(\mathrm{LBD})}$ were constructed to explore the neural underpinnings of LA by highlighting the structures that were specifically affected in one or the other of the two groups or that were damaged in both groups. Although there was a large region of lesion overlap between the two groups, subtraction of the superimposed lesions in LA+ ${ }_{(\mathrm{LBD})}$ and LA- ${ }_{\text {(LBD })}$ indicated that LA was associated with frontal and parietal lesions (Fig. 2). The across-groups differences were analyzed by means of Mann-Whitney tests performed on the following: (1) inferior parietal cortex (IPC), which comprises three anatomical structures of the inferior parietal cortex: supramarginal gyrus, angular gyrus, and a third region located above the supramarginal gyrus and between the supramarginal and angular gyri (Tzourio-Mazoyer et al., 2002); and (2) the inferior frontal gyrus (IFG), which consists of three macroscopic anatomical structures: pars opercularis, triangularis, and orbitalis (Amunts et al., 1999). The results show a significant difference for both IFG ( $p=$ $0.047)$ and IPC $(p=0.035)$. tasks

\section{Analysis of correct responses}

The accuracy with which the three groups judged whether the model correctly performed the different gestures were compared by a mixed-model ANOVA, with group $\left(\mathrm{LA}+_{(\mathrm{LBD})}, \mathrm{LA}-_{(\mathrm{LBD})}\right.$, LA- $\left.{ }_{(\mathrm{RBD})}\right)$ as between-subjects factor and task as within-subjects factor [two levels: recognition of correct execution of transitive (TGR) and intransitive (IGR) gestures performed by others]. The significant main effect of group $\left(F_{(2,38)}=18.48 ; p<0.0001\right)$ is 


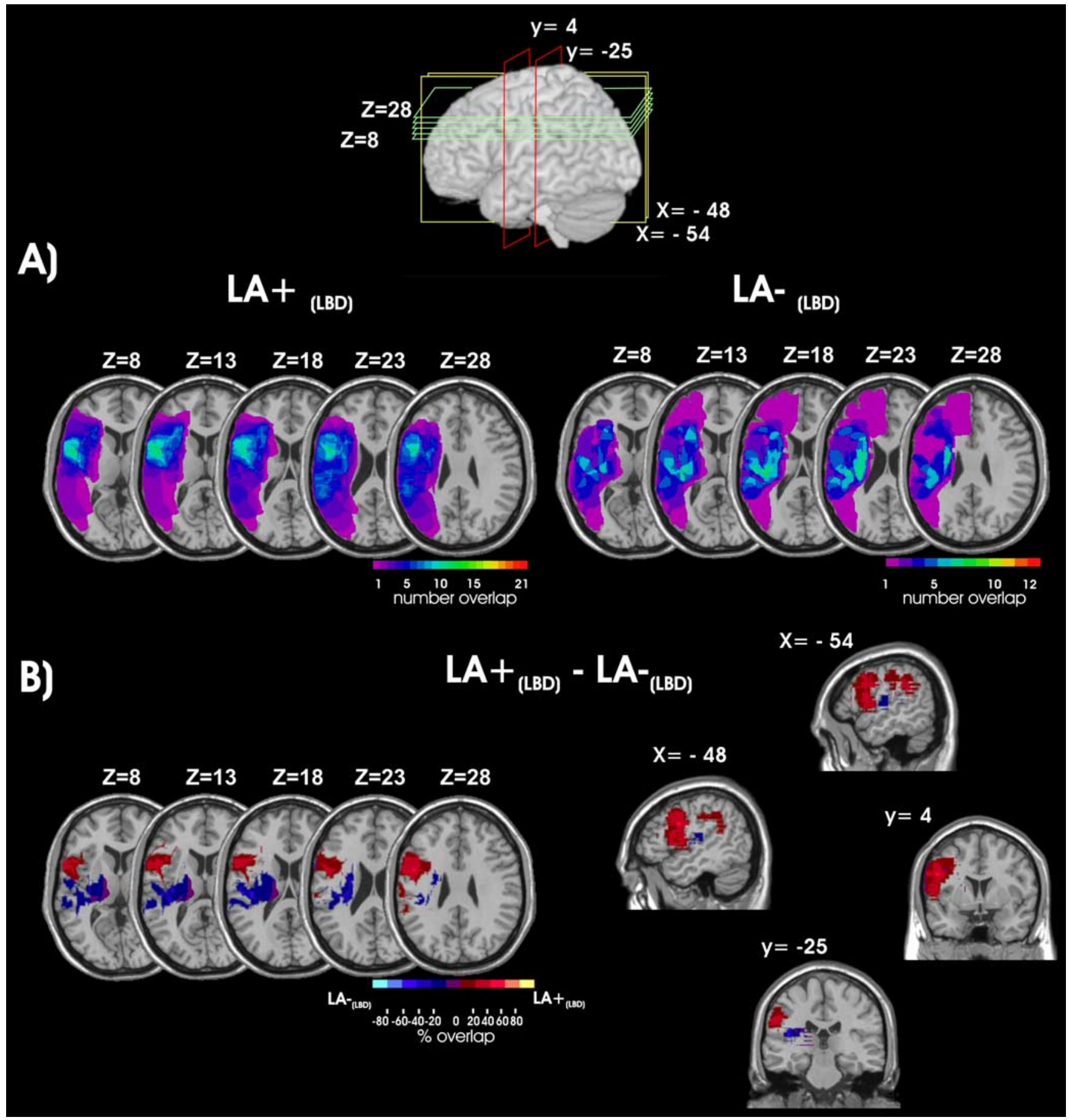

Figure 2. Lesion mapping in LBD patients. A, Overlays of regional lesion plots of the 21 patients with limb apraxia and gesture recognition deficit $\left(\mathrm{LA}+{ }_{(\mathrm{LBD})}\right)$ and of the 12 patients without limb apraxia $\left(L A-{ }_{(L B D)}\right)$. Legend, The number of overlapping lesions is illustrated by different colors that code for increasing frequencies from violet (lesion in one patient) to red (lesion in all the patients

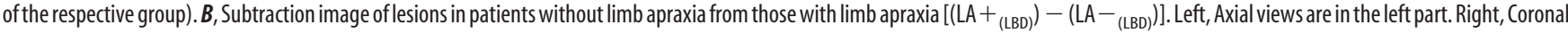
views and sagittal renderings. Legend, Lesion subtractions show the highest difference in lesion density illustrated by different colors that code for increasing frequencies from dark red to yellow (positive values) and from dark blue to light blue (negative values). Each color represents a $20 \%$ increment. Positive and negative values indicate regions damaged more frequently in patients with or without limb apraxia, respectively. The images show that limb apraxia is associated with frontoparietal lesions.

explained by the higher impairment of apraxic patients compared with nonapraxic patients (correct responses, LA+ ${ }_{(\mathrm{LBD})}$, $\left.20.45 ; \mathrm{LA}^{(\mathrm{LBD})}, 27.12 ; \mathrm{LA}-_{(\mathrm{RBD})}, 26.69\right)$. The significant main effect of task $\left(F_{(1,38)}=7.29 ; p<0.01\right)$ is attributable to the higher accuracy observed in the TGR (24.51) than in the IGR (22.7). This effect may be attributable to the fact that objects may provide unequivocal cues as to which action should be performed. In contrast, correct recognition of non-object-related gestures may require a first step of analysis in which subjects have to infer which gesture is intended, and a second step in which subjects have to judge whether the actor's intention is correctly executed. This additional need for inferential reasoning may in principle contribute to any deficits that may be specifically found in apraxic patients. However, the group by task interaction was not signifi- 
cant, indicating that apraxic patients were more impaired than the control patients in both object and non-object-related gestures. Moreover, when gesture execution (as assessed with IA and IMA tests) was correlated with gesture comprehension (TGR and IGR), significant positive correlations were observed for both the combined (mean combined IA and IMA score vs mean combined TGR and IGR score, $\left.r_{(41)}=0.83, p<0.0001\right)$ and the separate (IMA vs IGR, $r_{(41)}=0.63, p<0.0001$; IMA vs TGR, $r_{(41)}=0.59$, $p<0.0001$; IA vs IGR, $r_{(41)}=0.51, p=0.001$; IA vs TGR, $r_{(41)}=$ $0.47, p=0.002$ ) scores, indicating a clear relationship between action production and understanding.

Because our recognition test included only meaningful gestures, we performed an additional correlational analysis that considered only the performance in the execution of the meaningful gestures in the IMA. Significant positive correlations were observed for both the combined (mean combined IA and IMA score vs mean combined TGR and IGR score, $r_{(41)}=0.64, p<0.0001$ ) and the separate (IMA vs IGR, $r_{(41)}=0.58, p<0.0001$; IMA vs TGR, $\left.r_{(41)}=0.53, p<0.0001\right)$ scores.

These results demonstrate a clear relationship between performing and understanding meaningful gestures.

\section{Cluster analysis}

To better characterize the gesture recognition deficit in the 21 apraxic patients $\left(\mathrm{LA}+_{(\mathrm{LBD})}\right)$, the raw data were subjected to a hierarchical cluster analysis in which the patients were sorted according to their performance in the TGR and IGR tasks. Cluster analysis identifies groups with minimal within-group variation and maximal between-group variation. According to this sorting process, $14 \mathrm{LA}_{(\mathrm{LBD})}$ patients (nos. 1-12, 16, and 21) presented with a severe gesture recognition deficit $\left(\mathrm{LA}+{ }_{(\mathrm{GRD}+)}\right.$ group) and $7 \mathrm{LA}+_{(\mathrm{LBD})}$ patients (13-15 and 17-20) presented with no deficit $\left(\mathrm{LA}+_{(\mathrm{GRD}-)}\right.$ group). The distribution of the different patients between the two groups is shown in Figure $3 A$.

\section{Gesture comprehension deficits in the two LA $+_{(\text {LBD })}$ subgroups}

The performance of the two LA ${ }_{(\mathrm{LBD})}$ subgroups in the different gesture recognition tasks is shown in Figure $3 B$.

\section{Analysis of the correct responses in the two LA $+_{(\text {LBD })}$ subgroups}

A mixed-model ANOVA was performed using subgroup $\left(\mathrm{LA}+{ }_{(\mathrm{GRD}+)}\right.$ and $\left.\mathrm{LA}+_{(\mathrm{GRD}-)}\right)$ as the between-subjects factor and task (two levels, TGR and IGR) as the within-subjects factor. The significant main effect of subgroup $\left(F_{(1,19)}=52.9 ; p<0.0001\right)$ was attributable to the better performance of $\mathrm{LA}+_{(\mathrm{GRD}-)}$ patients (25.57) relative to $\mathrm{LA}{ }_{(\mathrm{GRD}+)}$ patients $(17.89)$. The significant main effect of task $\left(F_{(1,19)}=8.79 ; p<0.008\right)$ was attributable to better performance in the TGR (21.62) than in the IGR (19.28). However, the subgroup by task interaction was not significant $\left(F_{(1,19)}=0.87 ; p=0.36\right)$.

\section{Signal detection analysis in the two $\mathrm{LA}+_{(\mathrm{LBD})}$ subgroups}

Based on the signal detection method, we used the proportions of hits and false alarms of each patient in the TGR and IGR tasks to compute the target sensitivity $\left(\mathrm{d}^{\prime}\right)$ and response bias $(\beta)$ indices. These values were analyzed using two separate mixed-model ANOVAs, one for each index, with subgroup $\left(\mathrm{LA}+{ }_{(\mathrm{GRD}+)}\right.$, $\left.\mathrm{LA}+_{(\mathrm{GRD}-)}\right)$ as the between-subjects factor and gesture recognition task (IGR vs TGR) as the within-subjects factor. Analysis of $\mathrm{d}^{\prime}$ revealed that the significant main effect of subgroup $\left(F_{(1,19)}=\right.$ 23.62; $p<0.0001)$ was attributable to the higher accuracy
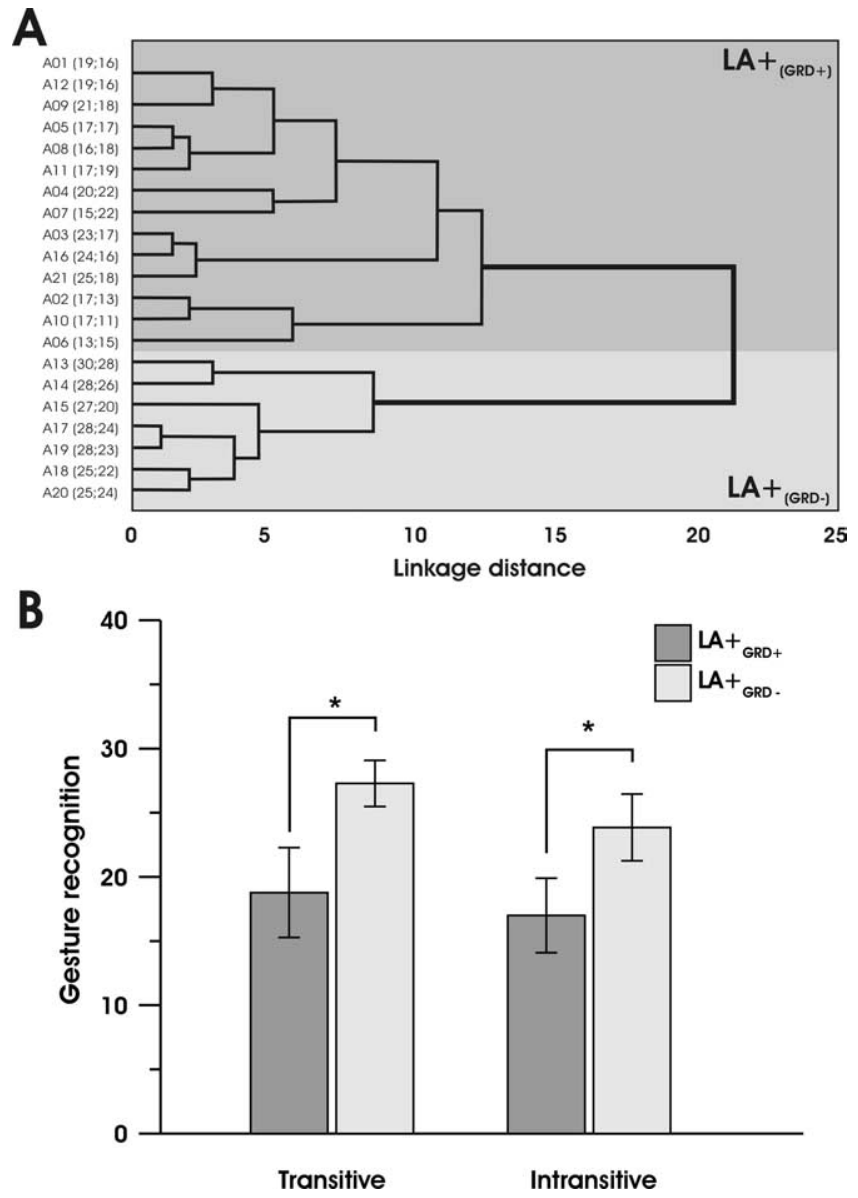

Figure 3. A, Dendrogram indicating the greatest difference between the performance of patients with limb apraxia $\left(\mathrm{LA}+{ }_{(\mathrm{LBD})}\right)$ in the two gesture recognition tasks. A complete-linkage hierarchical clustering algorithm was used. The $x$-axis shows Euclidean distances that provide a measure of $L A+{ }_{(\mathrm{BBD})}$ patients' performance similarity in TGR and IGR. The performance in the two gesture recognition tasks is reported along the $y$-axis. Patients with the most similar performance are closer to each other. Two main clusters are apparent. Patients with $\left(\mathrm{LA}+{ }_{(\mathrm{GRD}+)}\right)$ or without $\left(\mathrm{LA}+_{(G R D-)}\right)$ gesture recognition deficits fall in the dark gray and light gray areas, respectively. $\boldsymbol{B}$, Performance of limb apraxia patients in the gestural comprehension task. Mean correct responses of $\mathrm{LA}+_{(\mathrm{GRD}+)}(n=14)$ and $\mathrm{LA}+_{(\mathrm{GRD}-)}(n=7)$ subgroups for the transitive and intransitive items of the gesture comprehension test. Error bars represent SEM. ${ }^{*} p<0.05$.

achieved by the $\mathrm{LA}+_{(\mathrm{GRD}-)}$ patients $(2.0)$ compared with the $\mathrm{LA}+_{(\mathrm{GRD}+)}$ patients $(0.52)$. The significant main effect of gesture recognition task $\left(F_{(1,19)}=13.66 ; p<0.001\right)$ was attributable to the higher sensitivity in the TGR (1.48) than in the IGR (0.84) task. However, the subgroup by gesture recognition task interaction was not significant. In contrast, the $\beta$ values did not differ across subgroups or task. Therefore, the results reflect a genuine deficit in gesture discrimination rather than a specific criterion of response.

None of the patients committed errors in the task of pointing to objects named by the examiner, thus ruling out the possibility that the deficits in the $\mathrm{LA}+_{(\mathrm{GRD}+)}$ patients in the judgment of correctness of transitive gestures were attributable to deficits in object identification per se. No significant differences were detected between the $\mathrm{LA}+_{(\mathrm{GRD}+)}$ and $\mathrm{LA}+_{(\mathrm{GRD}-)}$ groups in the Raven (19.86 vs $\left.23.7 ; t_{(19)}=-1.82, p=0.08\right)$ and in the Token ( 37.5 vs $\left.46.7 ; t_{(19)}=-1.63, p=0.11\right)$ tests.

To further assess whether language comprehension influenced the gesture recognition task, we performed an analysis of covariance on the TGR and IGR data with scores in the token 
Table 1. Statistical comparisons of the lesioned areas in LBD patients with apraxia and gestural comprehension deficits (LA+ $\left.{ }_{(G R D+)}\right)$, with apraxia and no gestural comprehension deficits $\left(\mathrm{LA}+_{(\mathrm{GRD}-)}\right)$, and without gesture comprehension deficits independently from the presence of apraxia

\begin{tabular}{|c|c|c|c|c|c|c|c|}
\hline \multirow[b]{2}{*}{ Anatomical area } & \multirow[b]{2}{*}{$\begin{array}{c}\begin{array}{c}\mathrm{LA}+ \\
(\mathrm{GRD}+) \\
n=14\end{array} \\
\end{array}$} & \multirow{2}{*}{\multicolumn{2}{|c|}{$\begin{array}{l}\text { Overall } \\
\text { regional } \\
\text { chi } \\
\text { square } \\
\text { (p value) }\end{array}$}} & \multirow[b]{2}{*}{$\begin{array}{c}\text { Mann-Whitney } \\
\text { voxel count } \\
\text { (p value) }\end{array}$} & \multirow[b]{2}{*}{$\begin{array}{c}\text { LA+ \& LA- } \\
\text { (GRD-) } \\
n=19\end{array}$} & \multirow[b]{2}{*}{$\begin{array}{l}\text { Overall } \\
\text { regional } \\
\text { chi } \\
\text { square } \\
\text { (p value) }\end{array}$} & \multirow[b]{2}{*}{$\begin{array}{l}\text { Mann-Whitney } \\
\text { voxel count } \\
\text { (p value) }\end{array}$} \\
\hline & & & & & & & \\
\hline Precentral & 14 & 4 & 0.047 & 0.07 & 14 & 0.11 & 0.001 \\
\hline Frontal_Sup & 9 & 1 & 0.089 & 0.13 & 3 & 0.013 & 0.025 \\
\hline Frontal_mid & 10 & 1 & 0.04 & 0.09 & 4 & 0.01 & 0.023 \\
\hline Frontal_Inf_Oper & 14 & 3 & 0.01 & 0.001 & 11 & 0.017 & 0.0002 \\
\hline Frontal_Inf_Tri & 13 & 1 & 0.0019 & 0.0003 & 7 & 0.004 & 0.002 \\
\hline Rolandic_Ōper & 11 & 6 & 0.84 & 0.8 & 16 & 0.97 & 0.523 \\
\hline Insula & 14 & 5 & 0.18 & 0.22 & 17 & 0.6 & 0.610 \\
\hline Postcentral & 11 & 7 & 0.5 & 0.86 & 18 & 0.39 & 0.985 \\
\hline Parietal Inf & 6 & 6 & 0.16 & 0.046 & 7 & 0.99 & 0.952 \\
\hline SupraMārginal & 5 & 6 & 0.089 & 0.25 & 10 & 0.54 & 0.562 \\
\hline Putamen & 6 & 2 & 0.87 & 0.69 & 9 & 0.92 & 0.662 \\
\hline Angular & 3 & 4 & 0.25 & 0.2 & 5 & 0.93 & 0.309 \\
\hline Heschl & 8 & 3 & 0.87 & 0.97 & 10 & 0.92 & 0.619 \\
\hline Temporal_Sup & 8 & 7 & 0.12 & 0.15 & 16 & 0.18 & 0.173 \\
\hline Temporal_Pole_Sup & 5 & 1 & 0.6 & 0.36 & 2 & 0.18 & 0.100 \\
\hline Temporal_Mid & 4 & 4 & 0.42 & 0.22 & 9 & 0.46 & 0.20 \\
\hline \multirow{3}{*}{$\begin{array}{l}\text { Voxel wise } \\
\text { chi square }\end{array}$} & \multicolumn{3}{|c|}{ BA 44} & \multicolumn{3}{|r|}{ BA 6} & \\
\hline & & & & & & & \\
\hline & \multicolumn{3}{|c|}{$\begin{array}{c}\text { IFGop }\left(\begin{array}{lll}-48 & 9 & 14\end{array}\right) \\
\text { p-value }=0.002\end{array}$} & \multicolumn{3}{|c|}{$\begin{array}{l}\text { dPMc }\left(\begin{array}{lll}-49 & 6 & 12\end{array}\right) \\
\text { p-value }=0.002\end{array}$} & \\
\hline
\end{tabular}

Columns 2 (black), 3 (blue), and 6 (red) report the number of patients in each group with lesion in a specific AAL region (column 1). For each AAL, overall regional $\chi^{2}$ analyses (with Yates' correction) were performed using these values and reported in columns 4 and 7 . The analysis on the number of damaged voxels for each AAL was assessed by means of Mann-Whitney $U$ tests (columns 5 and 8 ). Significant comparisons are shown highlighted in gray. The Bonferroni correction for multiple comparisons was applied by taking into account the number of AAL regions. Therefore, the statistical threshold for significance was set at $p<0.0031$. The bottom panel shows Talairach coordinates of the lesioned voxels significantly associated with gesture recognition deficits: $x=-48, y=9$, and $z=14$ for the pars opercularis, corresponding to Brodmann's area 44 in the left inferior frontal gyrus (IFGop); $x=-49, y=6$, and $z=12$ for the premotor cortex, corresponding to Brodmann's area 6 in the dPMc.

comprehension test as a covariate and subgroup as a betweensubjects factor $\left(\mathrm{LA}+_{(\mathrm{GRD}+)}\right.$ and $\left.\mathrm{LA}+_{(\mathrm{GRD}-)}\right)$. Subgroup differences in gesture recognition remained highly significant, even when controlling for the token test scores $(F=42.37 ; p=$ $0.0001)$. The subgroup by language comprehension interaction was not significant, indicating that language comprehension deficits did not differ between the two LA+ subgroups. This rules out that the recognition of the correct execution of gestures performed by a model was primarily influenced by language comprehension.

\section{Anatomical analysis}

To determine whether any particular subregion within the common lesioned area was specific to the deficits observed in the gesture recognition task, we constructed lesion maps for LBD patients only.
Anatomical analysis in the two $\mathrm{LA}+_{(\mathrm{LBD})}$ subgroups

A lesion density image was generated for the two subgroups of LA+ patients with (Fig. $4 A$, left part) or without gesture recognition deficit (Fig. $4 A$, right part). Lesion subtractions in apraxic patients with and without gesture recognition deficits (Fig. $4 B$, axial, sagittal, and coronal views in the right and left parts) revealed that, whereas the dorsal premotor cortex (dPMc), the IFG, and adjacent insular cortex were more frequently damaged in $\mathrm{LA}+_{(\mathrm{GRD}+)}$, the supramarginal gyrus, the inferior parietal lobe, and the underlying parietal white matter were more frequently damaged in $\mathrm{LA}+_{(\mathrm{GRD}-)}$. The $\mathrm{LA}+_{(\mathrm{GRD}+)}$ and $\mathrm{LA}+_{(\mathrm{GRD}-)}$ subgroups did not differ in overall lesion volume (33.50 vs 40.72 $\mathrm{cm}^{3}$; Mann-Whitney $\left.U=48.0 ; Z=0.07 ; p=0.94\right)$.

We also examined in the two LA+ ${ }_{(\mathrm{LBD})}$ subgroups the possible influence of lesion size on gesture production or comprehension. No significant correlation between lesion volume and each of four gesture production or gesture comprehension tasks was 


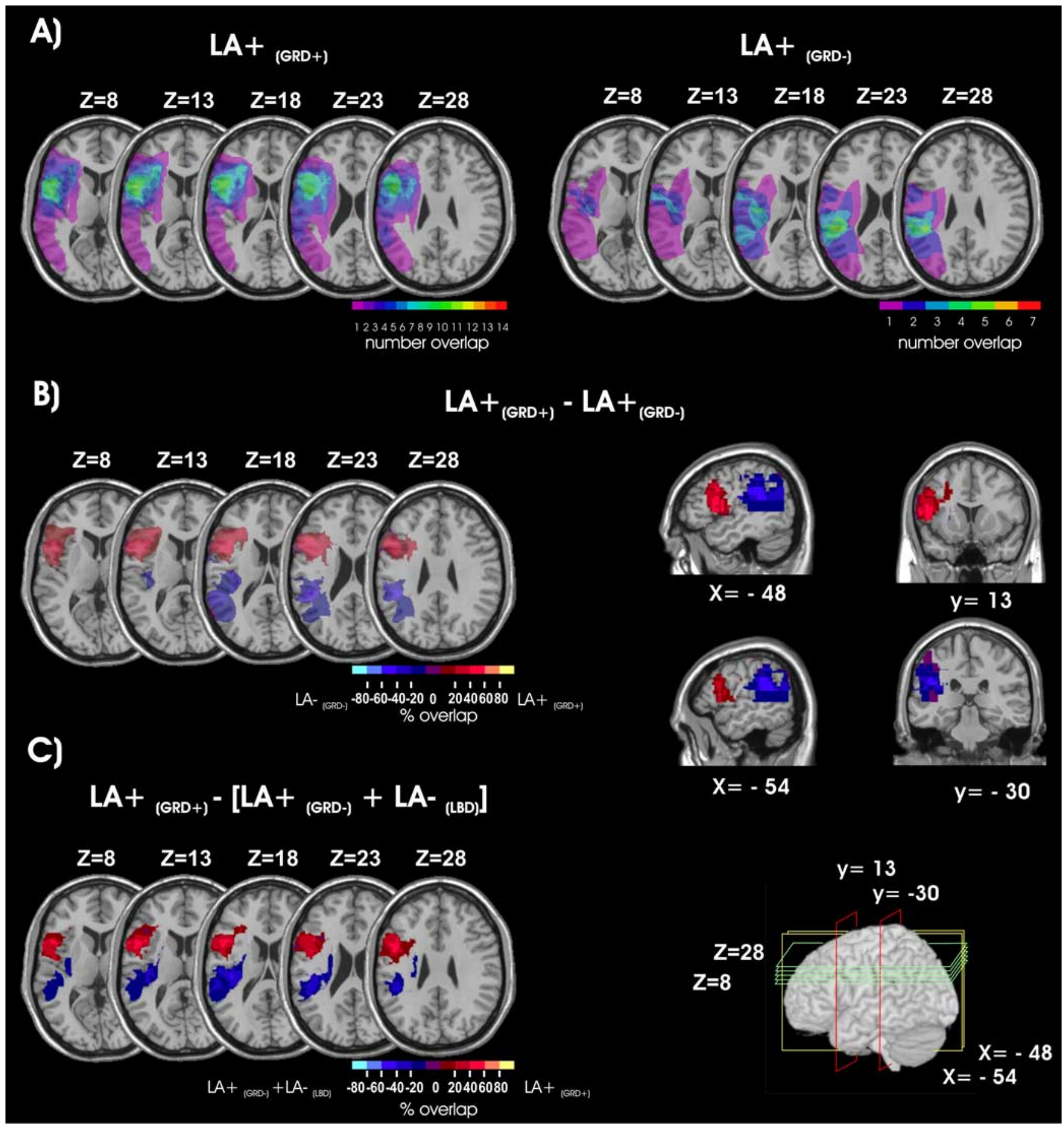

Figure 4. Anatomical underpinnings of gestural discrimination deficits. $A$, Axial views of regional lesion plots overlays in patients with limb apraxia with $\left(\mathrm{LA}+{ }_{(\mathrm{GRD}+)} ; n=14\right)$ or without gesture recognition deficit $\left(\mathrm{LA}+_{(\mathrm{GRD}-)} ; n=7\right)$. The legend is as for Figure $2 A$. B. Axial views (left) and coronal views and sagittal renderings (right) of overlay plots resulting from the subtraction of the proportional values of patients with $\left(\mathrm{LA}+_{(G R D+)}\right)$ or without $\left(\mathrm{LA}+_{(G R D-}\right)$ gesture recognition deficits. The legend is as for Figure $2 B$. Positive and negative values indicate regions damaged more frequently in $L A$ patients with and without gesture comprehension, respectively. $C$, Right, Proportional values subtraction of lesions of patients with no gestural recognition deficits $\left(L A+{ }_{(G R D-)}\right.$ plus $\left.\mathrm{LA}-{ }_{(\mathrm{LBD})}\right)$ from the lesion of patients with gesture recognition deficits $\mathrm{LA}+_{(\mathrm{GRD}+)}$. Left, Brain rendering showing the $x, y$, and $z$ planes at the different lesion cuts.

found (Spearman, $r_{\mathrm{s}(21)}=0.008, r_{\mathrm{s}(21)}=0.09, r_{\mathrm{s}(21)}=-0.17$, and $r_{\mathrm{s}(21)}=-0.16$ for IA, IMA, TGR, and IGR, respectively). This indicates that the overall lesion volume per se does not predict execution or recognition deficits.

Anatomical analysis in the two $\mathrm{LA}+_{(\mathrm{GRD}+)}$ and $\left(\mathrm{LA}+{ }_{(\mathrm{GRD}-)}\right.$ and $\left.\mathrm{LA}-{ }_{(\mathrm{LBD})}\right)$ subgroups

We explored the neural substrates of gestural recognition per se by subtracting from the lesions of $\mathrm{LA}+_{(\mathrm{GRD}+)}$ patients, the le- sions of patients without gesture comprehension deficit in whom limb apraxia might be either present or absent $\left(\mathrm{LA}-{ }_{(\mathrm{LBD})}\right.$ plus $\left.\mathrm{LA}+_{(\mathrm{GRD}-)}\right)($ Fig. 4C). Maximal lesion density differences confirmed that gesture recognition deficits were associated with frontal and insular lesions.

The $\mathrm{LA}+_{(\mathrm{GRD}+)}$ and $\left(\mathrm{LA}+_{(\mathrm{GRD}-)}\right.$ and $\left.\mathrm{LA}-_{(\mathrm{LBD})}\right)$ subgroups did not differ in overall lesion volume $\left(33.50 \mathrm{vs} 36.62 \mathrm{~cm}^{3}\right.$; Mann-Whitney $U=129.0 ; Z=0.14 ; p=0.88$ ). To further assess whether language comprehension influenced the gesture 
recognition task, we performed an ANOVA between the $\mathrm{LA}+{ }_{(\mathrm{GRD}+)}$ and LBD patients without gesture comprehension deficit $\left(\mathrm{LA}{ }_{(\mathrm{GRD}-)}\right.$ and $\left.\mathrm{LA}-{ }_{(\mathrm{LBD})}\right)$. Patients $\mathrm{LA}+_{(\mathrm{GRD}+)}$ have poorer comprehension than LA- ${ }_{\text {(LBD) }}$ patients. Hence, it is not surprising that a significant difference exists between groups ( 37.5 vs $\left.52.7 ; t_{(31)}=-3.58 ; p=0.001\right)$. We performed an ANCOVA on the gesture recognition scores of the two groups $\left[\mathrm{LA}+_{(\mathrm{GRD}+)}\right.$ and $\left(\mathrm{LA}+_{(\mathrm{GRD}-)}\right.$ and $\left.\left.\mathrm{LA}-_{(\mathrm{LBD})}\right)\right]$ with language comprehension scores as a covariate. There was no significant interaction $\left(F_{(1,29)}=0.50 ; p=0.48\right)$, indicating that gesture recognition deficit is not explained by the language comprehension deficit.

To test the influence of lesion size in specific cortical regions, we computed correlations (Pearson) between performance in the recognition and in execution of meaningful gestures in patients with damage to the inferior frontal gyrus or the inferior parietal cortex [according to the Tzourio-Mazoyer et al. (2002) subdivision] but not to both. The results of this analysis showed a significant correlation $\left(R_{(11)}=0.68 ; p<0.019\right)$ in the group of patients with lesions in the inferior frontal gyrus but not in the group of patients with parietal lesions $\left(R_{(6)}=0.60 ; p=0.2\right)$. The two subgroups did not differ with regard to overall lesion volume (38.90 vs $37.56 \mathrm{~cm}^{3}$; Mann-Whitney $U=23.50 ; Z=0.95 ; p=$ $0.34)$. Therefore, the results of these correlational analyses indicate that lesions to the inferior frontal gyrus affect the performance in both gesture recognition and execution independently from the presence of parietal damage.

To provide quantitative estimates of the differences observed in lesion subtractions and to determine the epicenter of the lesion related to gesture recognition deficits, we conducted a regional statistical analysis on the output of the AAL (Table 1).

Lesions associated with gesture comprehension deficits primarily involved the frontal agranular cortex, including the posterior pars opercularis of the IFG (corresponding to Brodmann's area 44/45, Talairach coordinates of the epicenter: $-48,9,14)$. In the gesture recognition task, the combined scores of TGR and IGR in apraxic patients with or without lesions to these voxels were 36.11 and 44.50, respectively (two-sample $t$ test, $t_{(19)}=2.46$; $p<0.02$ ). Although belonging to a region that was not significant in the omnibus tests the dorsal premotor cortex (corresponding to Brodmann's area 6, Talairach coordinates of the epicenter: $-49,6,12$ ) was significantly correlated with gesture recognition deficits.

We performed a correlational analysis between language comprehension scores obtained in token test and the percentage of lesioned voxel in IFG. No significant correlation was found when considering the 21 apraxic patients (Spearman, $r_{\mathrm{s}(21)}=0.29$ ) or the 33 LBD patients $\left(r_{\mathrm{s}(33)}=0.12\right)$.

Importantly, we performed Spearman rank correlations analyses to test the relationship between percentage of lesion voxels in the different lesioned AAL areas and the scores in the gesture recognition task. Significant negative correlations between the left IFG lesions and the TGR and IGR gestures $\left(r_{\mathrm{s}(21)}=-0.57\right.$, $p<0.005$ for TGR; $r_{\mathrm{s}(21)}=-0.59, p<0.0004$ for IGR) are shown in Figure 5.

We further analyzed this effect by capitalizing on the notion that the human IFG consists of three macroscopic anatomical structures: pars opercularis, triangularis, and orbitalis. Separate Spearman rank correlation analyses performed for both the pars opercularis $\left(r_{\mathrm{s}(21)}=-0.49, p<0.02\right.$ for TGR; $r_{\mathrm{s}(21)}=-0.54, p<$ 0.01 for IGR) and the triangularis $\left(r_{\mathrm{s}(21)}=-0.69, p<0.0004\right.$ for TGR; $\left.r_{\mathrm{s}(21)}=-0.65, p<0.001\right)$ revealed significant negative correlations. In contrast, there was no significant correlation with
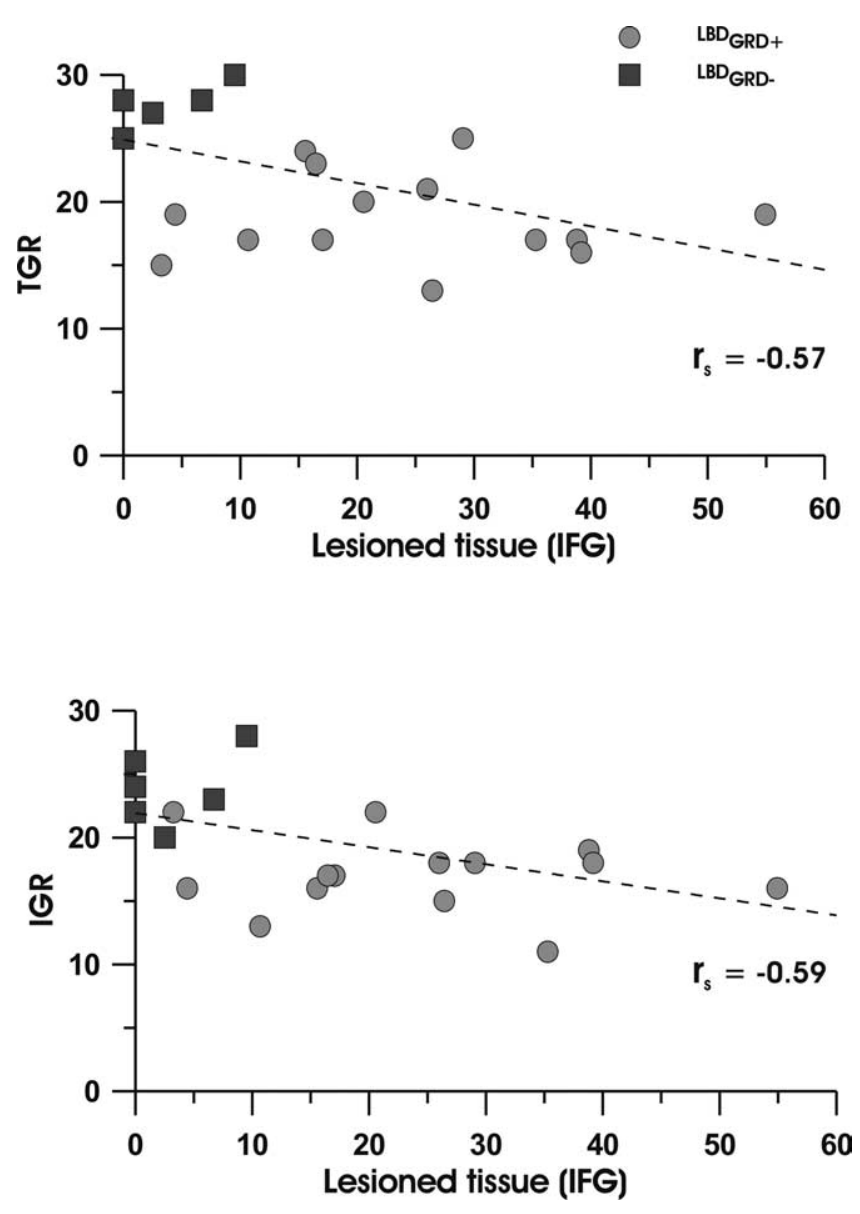

Figure 5. Scatter plots of the TGR and IGR scores ( $y$-axis) versus the percentage of IFG lesion voxels $\left(x\right.$-axis) in patients with limb apraxia, with $\left(\mathrm{LA}+_{(\mathrm{GRD}+)}\right.$; filled circles) or without gesture recognition deficit (LA+ ${ }_{(\mathrm{GRD}-)}$; filled squares).

lesions in pars orbitalis or in any other areas. Thus, gesture comprehension deficits were associated with lesions of the IFG, pars opercularis and triangularis. Interestingly, these two regions appear to be involved in imitation (Iacoboni et al., 1999; Koski et al., 2002; Muhlau et al., 2005; Goldenberg and Karnath, 2006; Molnar-Szakacs et al., 2006) and execution of object-directed hand actions (Binkofski et al., 1999) as well as in observation of visually presented actions and linguistic phrases describing actions (Aziz-Zadeh et al., 2006).

The two anatomical maps shown in Figure 6, obtained by means of the nonparametric permuted Brunner-Munzel rankorder statistic analysis, indicate the lesioned voxels that significantly impact on the gesture comprehension tasks. It can be seen that a focus centered on the inferior frontal gyrus and Rolandic operculum (corresponding to Brodmann areas 44 and 45 and extending into area 6) seems to be causatively associated to gesture recognition deficits (Fig. 6A).

The causal relationship between damage to these regions and gesture recognition have predictive value even after overall lesion volume has been covaried out (Fig. 6B).

\section{Discussion}

We assessed the performance of brain-damaged patients with or without LA by using a novel ad hoc test that explored their ability to judge the correct execution of gestures performed by a model. We also explored the neural underpinnings of gestural comprehension by correlating deficits exhibited in the above test with the 
site and extent of the brain lesions. We found that (1) apraxic patients who, by definition, are impaired in performing actions, were also impaired in judging the execution of gestures performed by others; and (2) lesions centered on the left inferior frontal cortex were specifically associated with gesture recognition deficits.

\section{Seeing and doing in apraxia}

Behavioral evidence indicates that observing a particular action facilitates the execution of the same action and inhibits actions that are different from those observed (Brass et al., 2000, 2001; Craighero et al., 2002). Moreover, neuroimaging and neurophysiological studies have shown that observing an action activates frontoparietal circuits (Iacoboni et al., 1999; Manthey et al., 2003; Johnson-Frey et al., 2005; Molnar-Szakacs et al., 2006) typically involved in the motor planning and execution of the same actions (Rizzolatti and Craighero, 2004). Although additional nonspecifically motoric cortical regions (e.g., extrastriate body area) (Costantini et al., 2005; Hamilton et al., 2006) and subcortical regions (e.g., the cerebellum) (Calvo-Merino et al., 2006) may be activated during mere action observation, neuroimaging studies in healthy subjects have suggested that action imitation and pantomime production are associated with the frontal and parietal cortices (Muhlau et al., 2005; Fridman et al., 2006). This motor mirroring process suggests that the observed actions are automatically mapped onto specific regions in the brain of the onlookers and may be fundamental for understanding what the observed agent is doing. Studies of brain-damaged patients with LA may help to clarify whether deficits in both imitative and nonimitative action execution parallel deficits in action recognition. Moreover, these studies offer a very good opportunity to identify neural regions that are causatively involved in both gesture execution and understanding. However, studies on this issue have provided controversial results (Heilman et al., 1982; Ferro et al., 1983; Rothi et al., 1985; Halsband et al., 2001; Buxbaum et al., 2005). Our finding indicated that apraxic patients were more impaired than nonapraxic patients not only in action execution but also in judging the correct execution of the observed action. This specific relationship was further supported by the significant positive correlation between deficits in gesture production and deficit in gesture comprehension. The parallel impairment in gesture execution and recognition suggests that the motor skills of apraxic patients may directly influence their visual action recognition. This is consistent with the novel notion of the mutual influence of sensory and motor components on action processing. Indeed, it is known that mere action observation may strengthen the motor representation of the observed action (Stefan et al., 2005), as well as mere motor experience of that particular action may improve its visual dis-
Voxel-based lesion mapping $Z=8 \quad Z=13 \quad Z=18 \quad Z=23 \quad Z=28 \quad Z=33$

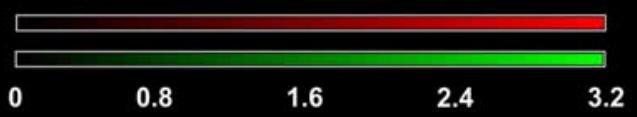

.2

[ Lesions associated with performance in TGR $p<0.05$ (FDR=2.66)

Lesions associated with performance in IGR $\mathrm{p}<\mathbf{0 . 0 5}$ ( $\mathrm{FDR}=\mathbf{2 . 4 8}$ )

Overlap between lesions associated with performance in TGR and IGR

Figure 6. Voxel-based lesion-symptom mapping for intransitive and transitive gesture recognition performance. $\boldsymbol{A}$, The mance in the group of 33 LBD patients on a voxel-by-voxel basis. The behavioral measures were the patients' accuracy in 列 both tasks are shown in red, green, and yellow, respectively. $\boldsymbol{B}$. Voxelwise logistic regression. This analysis was computed for all the voxels, with overall lesion volume as a covariate. All the voxels that exceed an uncorrected $p<0.05$ are shown. The colors correspond to the Z-score, with yellow regions indicating regions that predict the presence of gesture recognition deficits.

crimination (Casile and Giese, 2006). This bidirectional influence between action observation and execution suggested to us to include both types of tasks in rehabilitation programs for LA (Smania et al., 2000; 2006). As posited by influential cognitive neuropsychological models of apraxia (Rothi et al., 1991; Cubelli et al., 2000) and demonstrated by clinical studies (Goldenberg and Hagmann, 1997; Cubelli et al., 2000; Bartolo et al., 2001; Rumiati et al., 2001; Tessari et al., 2007), the range of possible dissociations between action execution and action understanding that can occur in apraxia is quite multifarious and cannot be explained by a mere action mirroring mechanism nor by a single lesion locus. Indeed, failures in imitating or in recognizing gestures may occur because of damage to a putative action semantics system or because of damage at any level in the process between perceiving (input lexicon) and performing (output lexicon) an action (Rothi et al., 1991; Cubelli et al., 2000). However, higherorder computations (e.g., related to intention to act, to distinct memory traces for different types of action, or to the ecological 
and cultural conditions in which an action is implemented) likely interact with low-level motor "resonance" mechanisms (e.g., the automatic selection of action primitives on which imitation and judgments regarding action appropriateness are based). Future studies addressing this outstanding link may help to understand the wide and complex range of human actions (meaningful vs meaningless, transitive vs intransitive, body- vs world-centered) in both normal and pathological conditions.

\section{A possible causative role for the left inferior frontal cortex in action understanding}

An important, still debated question concerns whether the mirroring process that likely allows action understanding, depends on purely motoric representations or whether it is related to visual inference and visual knowledge. Relevant to this issue is the fMRI study of ballet dancers demonstrating that neural activation in nonvisual regions is contingent on action observation, thereby indicating a purely motor influence on visual expertise (Calvo-Merino et al., 2006). Importantly, studies based on the effect of temporary virtual lesions induced by repetitive transcranial magnetic stimulation demonstrated that the inferior frontal cortex is crucial for action understanding (Pobric and Hamilton, 2006), pure visual discrimination of actions (Urgesi et al., 2007), and imitation (Heiser et al., 2003). Interestingly, a monkey fMRI study showed that the frontal lobe hosts multiple representations of others' actions and that only area F5a (Nelissen et al., 2005), a region considered to be the monkey homolog of human BA 44 (Petrides and Pandya, 2002; Petrides et al., 2005), represents the observed actions in an abstract, context-independent manner.

In keeping with recent anatomical studies (Haaland et al., 2000), our results showed that the frontal and parietal lesions were comparable in apraxic patients, indicating the importance of both these areas in performing gestures. A novel result of the present study is that impairments in the recognition of both transitive and intransitive gestures performed by a model were greater in apraxic patients with frontal lesions. Although apraxic patients also presented with parietal damage, the gesture comprehension deficits were not associated with lesions to this area. This pattern of results may hint at a key causative role of the left frontal cortex in gesture understanding. Our findings of a comparatively minor influence of parietal lesions on gesture recognition may be surprising, because the cortical network activated during action observation and execution involves both frontal and parietal nodes (Iacoboni et al., 1999, 2005; Manthey et al., 2003; Johnson-Frey et al., 2005; MolnarSzakacs et al., 2006). Although the few previous studies that directly addressed this issue in brain-damaged patients have emphasized the predominant role of the parietal lobe (Heilman et al., 1982; Rothi et al., 1985; Buxbaum et al., 2005), no definite conclusion could be drawn because most subjects appeared to have lesions involving both frontal and parietal lobes (Buxbaum et al., 2005) and/or radiological images were absent (Sirigu et al., 1995). Moreover, a study involving a comparatively large sample of left or right brain-damaged patients with lesions centered in the parietal or the premotor cortices failed to show any relationship between action execution and comprehension (Halsband et al., 2001). The only research performed to date using advanced lesionreconstruction techniques, however, suggested that the parietal lobe may be crucial for recognizing pantomime gestures (Buxbaum et al., 2005). Relevant to our finding is a recent lesion mapping study that showed that deficits in pantomime are specifically associated with lesions of the IFG, adjacent portions of the insula, and the precentral gyrus, but not with parietal lesions (Goldenberg et al., 2007).

To our knowledge, our study is the first to demonstrate that frontal structures have a prominent role in the ability of apraxic patients to recognize gestures performed by others; this finding may stand in contrast to the previous findings that suggest a major role of the parietal structures in gesture understanding (Heilman et al., 1982; Rothi et al., 1985; Buxbaum et al., 2005). This discrepancy may be explained by the following two lines of evidence that are discussed in the order of increasing relevance. First, whereas our gesture comprehension test required few or no verbal demands, almost all the gesture comprehension tests used in previous studies were inherently verbal. Therefore, our results may be in agreement with previous results showing that aphasic patients with lesions centered on the inferior frontal cortex presented with deficits in the nonlinguistic interpretation of actions, whereas aphasic patients with lesions centered in the temporal and parietal structures were impaired in comparable tasks with strong linguistic components (Saygin et al., 2004). The second, and possibly the most important, line of evidence is that our gesture recognition task differed from those used previously because the errors of the model did not affect the purely executive components of the gestures. Apraxic patients with parietal lesions are more impaired in executing transitive than intransitive gestures (Sirigu et al., 1995; Dumont et al., 1999; Buxbaum et al., 2005). Moreover, studies on both action execution and action observation indicate that the parietal lobe may be preferentially engaged when the kinematics and afferent components of the action are implemented or simulated (Sirigu et al., 1995; Sunderland and Sluman, 2000; Bosbach et al., 2005; Buxbaum et al., 2005; Costantini et al., 2005). In all these studies, however, changes in the hand posture kinematics were higher in transitive than in intransitive gestures. In our gesture comprehension test, the kinematic-proprioceptive features and the general plan of action were maintained even in the incorrect gestures, for both transitive and intransitive gestures. For example, the same typical movement for cutting bread with a knife was also used when attempting to cut the bread with a spoon. In a similar vein, the kinematics of the hitchhiking sign was preserved even when performed in an erroneous spatial position. Thus, it is plausible that our task assessed the ability to judge whether the ultimate goals of transitive gestures are attained or whether the symbolic meaning of intransitive gestures is maintained. This difference may explain the comparatively minor involvement of the parietal lobe in our task. Thus, although our results do not rule out the importance of the parietal cortex in gesture comprehension, they indicate the complex nature of gesture understanding that, similar to action planning and execution, should be regarded as a multicomponential process. In this vein, it is overly simplistic to consider that a single lesional locus is responsible for all possible types of gesture recognition deficits.

\section{References}

Amunts K, Schleicher A, Burgel U, Mohlberg H, Uylings HB, Zilles K (1999) Broca's region revisited: cytoarchitecture and intersubject variability. J Comp Neurol 412:319-341.

Aziz-Zadeh L, Wilson SM, Rizzolatti G, Iacoboni M (2006) Congruent embodied representations for visually presented actions and linguistic phrases describing actions. Curr Biol 16:1818-1823. 
Bartolo A, Cubelli R, Della Sala S, Drei S, Marchetti C (2001) Double dissociation between meaningful and meaningless gesture reproduction in apraxia. Cortex 37:696-699.

Binkofski F, Buccino G, Posse S, Seitz RJ, Rizzolatti G, Freund H (1999) A fronto-parietal circuit for object manipulation in man: evidence from an fMRI-study. Eur J Neurosci 11:3276-3286.

Bosbach S, Cole J, Prinz W, Knoblich G (2005) Inferring another's expectation from action: the role of peripheral sensation. Nat Neurosci 8:1295-1297.

Brass M, Bekkering H, Wohlschlager A, Prinz W (2000) Compatibility between observed and executed finger movements: comparing symbolic, spatial, and imitative cues. Brain Cogn 44:124-143.

Brass M, Zysset S, von Cramon DY (2001) The inhibition of imitative response tendencies. NeuroImage 14:1416-1423.

Buxbaum LJ, Kyle KM, Menon R (2005) On beyond mirror neurons: internal representations subserving imitation and recognition of skilled object-related actions in humans. Brain Res Cogn Brain Res 25:226-239.

Calvo-Merino B, Grezes J, Glaser DE, Passingham RE, Haggard P (2006) Seeing or doing? Influence of visual and motor familiarity in action observation. Curr Biol 16:1905-1910.

Casile A, Giese MA (2006) Nonvisual motor training influences biological motion perception. Curr Biol 16:69-74.

Costantini M, Galati G, Ferretti A, Caulo M, Tartaro A, Romani GL, Aglioti SM (2005) Neural systems underlying observation of humanly impossible movements: an FMRI study. Cereb Cortex 15:1761-1767.

Craighero L, Bello A, Fadiga L, Rizzolatti G (2002) Hand action preparation influences the responses to hand pictures. Neuropsychologia 40:492-502.

Cubelli R, Marchetti C, Boscolo G, Della Sala S (2000) Cognition in action: testing a model of limb apraxia. Brain Cogn 44:144-165.

Daprati E, Sirigu A (2006) How we interact with objects: learning from brain lesions. Trends Cogn Sci 10:265-270.

De Renzi E, Lucchelli F (1988) Ideational apraxia. Brain 111:1173-1185.

De Renzi E, Motti F, Nichelli P (1980) Imitating gestures. A quantitative approach to ideomotor apraxia. Arch Neurol 37:6-10.

Dumont C, Ska B, Schiavetto A (1999) Selective impairment of transitive gestures: an unusual case of apraxia. Neurocase 5:447-458.

Ferro JM, Martins IP, Mariano G, Caldas AC (1983) CT scan correlates of gesture recognition. J Neurol Neurosurg Psychiatry 46:943-952.

Fogassi L, Ferrari PF, Gesierich B, Rozzi S, Chersi F, Rizzolatti G (2005) Parietal lobe: from action organization to intention understanding. Science 308:662-667.

Fridman EA, Immisch I, Hanakawa T, Bohlhalter S, Waldvogel D, Kansaku K, Wheaton L, Wu T, Hallett M (2006) The role of the dorsal stream for gesture production. NeuroImage 29:417-428.

Gallese V, Fadiga L, Fogassi L, Rizzolatti G (1996) Action recognition in the premotor cortex. Brain 119:593-609.

Goldenberg G, Hagmann S (1997) The meaning of meaningless gestures: a study of visuo-imitative apraxia. Neuropsychologia 35:333-341.

Goldenberg G, Karnath HO (2006) The neural basis of imitation is body part specific. J Neurosci 26:6282-6287.

Goldenberg G, Hermsdorfer J, Glindemann R, Rorden C, Karnath HO (2007) Pantomime of tool use depends on integrity of left inferior frontal cortex. Cereb Cortex 17:2769-2776

Green DM, Swets JA (1966) Signal detection theory and psychophysics. New York: Wiley.

Haaland KY, Harrington DL, Knight RT (2000) Neural representations of skilled movement. Brain 123:2306-2313.

Halsband U, Schmitt J, Weyers M, Binkofski F, Grutzner G, Freund HJ (2001) Recognition and imitation of pantomimed motor acts after unilateral parietal and premotor lesions: a perspective on apraxia. Neuropsychologia 39:200-216.

Hamilton AF, Wolpert DM, Frith U, Grafton ST (2006) Where does your own action influence your perception of another person's action in the brain? NeuroImage 29:524-535.

Hanna-Pladdy B, Heilman KM, Foundas AL (2001) Cortical and subcortical contributions to ideomotor apraxia: analysis of task demands and error types. Brain 124:2513-2527.

Heilman KM, Rothi LJ, Valenstein E (1982) Two forms of ideomotor apraxia. Neurology 32:342-346.

Heiser M, Iacoboni M, Maeda F, Marcus J, Mazziotta JC (2003) The essential role of Broca's area in imitation. Eur J Neurosci 17:1123-1128.
Iacoboni M, Woods RP, Brass M, Bekkering H, Mazziotta JC, Rizzolatti G (1999) Cortical mechanisms of human imitation. Science 286:2526-2528.

Iacoboni M, Molnar-Szakacs I, Gallese V, Buccino G, Mazziotta JC, Rizzolatti G (2005) Grasping the intentions of others with one's own mirror neuron system. PLoS Biol 3:0001-0007.

Johnson-Frey SH, Newman-Norlund R, Grafton ST (2005) A distributed left hemisphere network active during planning of everyday tool use skills. Cereb Cortex 15:681-695.

Koski L, Wohlschlager A, Bekkering H, Woods RP, Dubeau MC, Mazziotta JC, Iacoboni M (2002) Modulation of motor and premotor activity during imitation of target-directed actions. Cereb Cortex 12:847-855.

Leiguarda RC (2005) Apraxias as traditionally defined. In: Higher-order motor disorders: from neuroanatomy and neurobiology to clinical neurology (Freund HJ, Jeannerod M, Hallet M, Leiguarda R, eds), pp 303337. New York: Oxford UP.

Leiguarda RC, Marsden CD (2000) Limb apraxias: higher-order disorders of sensorimotor integration. Brain 123:860-879.

Luzzatti C, Willmes K, De Bleser R (1996) Aachener Aphasie Test (AAT). Versione Italiana. Florence, Italy: Organizzazioni Speciali.

Manthey S, Schubotz RI, von Cramon DY (2003) Premotor cortex in observing erroneous action: an fMRI study. Brain Res Cogn Brain Res 15:296-307.

McClain M, Foundas A (2004) Apraxia. Curr Neurol Neurosci Rep 4:471-476.

Molnar-Szakacs I, Kaplan J, Greenfield PM, Iacoboni M (2006) Observing complex action sequences: the role of the fronto-parietal mirror neuron system. NeuroImage 33:923-935.

Muhlau M, Hermsdorfer J, Goldenberg G, Wohlschlager AM, Castrop F, Stahl R, Rottinger M, Erhard P, Haslinger B, Ceballos-Baumann AO, Conrad B, Boecker H (2005) Left inferior parietal dominance in gesture imitation: an fMRI study. Neuropsychologia 43:1086-1098.

Nelissen K, Luppino G, Vanduffel W, Rizzolatti G, Orban GA (2005) Observing others: multiple action representation in the frontal lobe. Science 310:332-336.

Nichols T, Hayasaka S (2003) Controlling the familywise error rate in functional neuroimaging: a comparative review. Stat Methods Med Res 12:419-446.

Oldfield RC (1971) The assessment and analysis of handedness: the Edinburgh inventory. Neuropsychologia 9:97-113.

Petrides M, Pandya DN (2002) Comparative architectonic analysis of the human and the macaque ventrolateral prefrontal cortex and corticocortical connection patterns in the monkey. Eur J Neurosci 16:291-310.

Petrides M, Cadoret G, Mackey S (2005) Orofacial somatomotor responses in the macaque monkey homologue of Broca's area. Nature 435:1235-1238.

Pizzamiglio L, Judica A, Razzano C, Zoccolotti P (1989) Toward a comprehensive diagnosis of visual-spatial disorders in unilateral brain damaged patients. Psychol Assess 5:199-218.

Pobric G, Hamilton AF (2006) Action understanding requires the left inferior frontal cortex. Curr Biol 16:524-529.

Raven JC, Court JH, Raven J (1988) Manual for Raven's progressive matrices and vocabulary scales. Oxford: Oxford Psychologists.

Rizzolatti G, Craighero L (2004) The mirror-neuron system. Annu Rev Neurosci 27:169-192.

Rorden C, Brett M (2000) Stereotaxic display of brain lesions. Behav Neurol 12:191-200.

Rorden C, Karnath HO (2004) Using human brain lesions to infer function: a relic from a past era in the fMRI age? Nat Rev Neurosci 5:813-819.

Rorden C, Karnath HO, Bonilha L (2007) Improving lesion-symptom mapping. J Cogn Neurosci 19:1081-1088.

Rothi LJ, Heilman KM, Watson RT (1985) Pantomime comprehension and ideomotor apraxia. J Neurol Neurosurg Psychiatry 48:207-210.

Rothi LJG, Ochipa C, Heilman KM (1991) A cognitive neuropsychological model of limb praxis. Cogn Neuropsychol 8:443-458.

Rumiati RI, Zanini S, Vorano L, Shallice T (2001) A form of ideational apraxia as a selective deficit of contention scheduling. Cogn Neuropsychol 18:617-642.

Saygin AP, Wilson SM, Dronkers NF, Bates E (2004) Action comprehension in aphasia: linguistic and non-linguistic deficits and their lesion correlates. Neuropsychologia 42:1788-1804. 
Sirigu A, Cohen L, Duhamel JR, Pillon B, Dubois B, Agid Y (1995) A selective impairment of hand posture for object utilization in apraxia. Cortex 31:41-55

Smania N, Girardi F, Domenicali C, Lora E, Aglioti S (2000) The rehabilitation of limb apraxia: a study in left-brain-damaged patients. Arch Phys Med Rehabil 81:379-388.

Smania N, Aglioti SM, Girardi F, Tinazzi M, Fiaschi A, Cosentino A, Corato E (2006) Rehabilitation of limb apraxia improves daily life activities in patients with stroke. Neurology 67:2050-2052.

Snodgrass JG, Vanderwart M (1980) A standardized set of 260 pictures: norms for name agreement, image agreement, familiarity, and visual complexity. J Exp Psychol [Hum Learn] 6:174-215.

Stefan K, Cohen LG, Duque J, Mazzocchio R, Celnik P, Sawaki L, Ungerleider L, Classen J (2005) Formation of a motor memory by action observation. J Neurosci 25:9339-9346.

Sunderland A, Sluman SM (2000) Ideomotor apraxia, visuomotor control and the explicit representation of posture. Neuropsychologia 38:923-934.
Talairach J, Tournoux P (1988) Co-planar stereotaxic atlas of the human brain. New York: Thieme.

Tessari A, Canessa N, Ukmar M, Rumiati RI (2007) Neuropsychological evidence for a strategic control of multiple routes in imitation. Brain 130:1111-1126.

Toraldo A, Reverberi C, Rumiati RI (2001) Critical dimensions affecting imitation performance of patients with ideomotor apraxia. Cortex 37:737-740.

Tzourio-Mazoyer N, Landeau B, Papathanassiou D, Crivello F, Etard O, Delcroix N, Mazoyer B, Joliot M (2002) Automated anatomical labeling of activations in SPM using a macroscopic anatomical parcellation of the MNI MRI single-subject brain. NeuroImage 15:273-289.

Urgesi C, Candidi M, Ionta S, Aglioti SM (2007) Representation of body identity and body actions in extrastriate body area and ventral premotor cortex. Nat Neurosci 10:30-31.

Watson RT, Fleet WS, Gonzalez-Rothi L, Heilman KM (1986) Apraxia and the supplementary motor area. Arch Neurol 43:787-792. 